\title{
Nonclassicality of Temporal Correlations
}

\author{
Stephen Brierley, ${ }^{1}$ Adrian Kosowski, ${ }^{2}$ Marcin Markiewicz, ${ }^{3, *}$ Tomasz Paterek, ${ }^{4,5,6}$ and Anna Przysiężna ${ }^{7,8}$ \\ ${ }^{1}$ DAMPT, Centre for Mathematical Sciences, University of Cambridge, Wilberforce Road, \\ Cambridge CB3 OWA, United Kingdom \\ ${ }^{2}$ Inria and Université Paris Diderot, LIAFA, Case 7014, 75205 Paris Cedex 13, France \\ ${ }^{3}$ Center for Theoretical Physics, Polish Academy of Sciences, Aleja Lotników 32/46, PL-02-668 Warszawa, Poland \\ ${ }^{4}$ School of Physical and Mathematical Sciences, Nanyang Technological University, 637371 Singapore, Singapore \\ ${ }^{5}$ Centre for Quantum Technologies, National University of Singapore, 117543 Singapore, Singapore \\ ${ }^{6}$ MajuLab, CNRS-UNS-NUS-NTU International Joint Research Unit, UMI 3654 Singapore, Singapore \\ ${ }^{7}$ Institute of Theoretical Physics and Astrophysics, University of Gdańsk, 80-952 Gdańsk, Poland \\ ${ }^{8}$ National Quantum Information Centre in Gdańsk, Andersa 27, 81-824 Sopot, Poland \\ (Received 4 March 2015; revised manuscript received 26 May 2015; published 18 September 2015)
}

\begin{abstract}
The results of spacelike separated measurements are independent of distant measurement settings, a property one might call two-way no-signaling. In contrast, timelike separated measurements are only one-way no-signaling since the past is independent of the future but not vice versa. For this reason some temporal correlations that are formally identical to nonclassical spatial correlations can still be modeled classically. We propose a new formulation of Bell's theorem for temporal correlations; namely, we define nonclassical temporal correlations as the ones which cannot be simulated by propagating in time the classical information content of a quantum system given by the Holevo bound. We first show that temporal correlations between results of any projective quantum measurements on a qubit can be simulated classically. Then we present a sequence of general measurements on a single $m$-level quantum system that cannot be explained by propagating in time an $m$-level classical system and using classical computers with unlimited memory.
\end{abstract}

DOI: 10.1103/PhysRevLett.115.120404

PACS numbers: 03.65.Ta, 03.65.Ud, 03.67.-a

Introduction.-The violation of a Bell inequality [1-3] demonstrates that the outcomes of an experiment have contradicted a set of well-defined classical intuitions. Quantum mechanics allows correlations between spacelike separated parties that have no explanation in terms of a hidden variable model; i.e., they cannot be reproduced with the help of classical computers running preagreed algorithms. However, when correlations are generated in a temporal scenario, by a sequence of timelike separated measurements, it is more difficult to demonstrate their nonclassical nature. The causal structure of physics implies only one-way no-signaling, namely the impossibility of sending communication backwards in time. The only bound on forward signaling is the information capacity of the physical system.

Here we analyze a single quantum system measured at $n$ points in time and consider to what extent one can prove that the temporal correlations between these measurement outcomes could not be generated by a classical system. We assume an idealization, in which the $m$-level physical system carries no hidden degrees of freedom. In this case the classical information capacity of the system is $\log _{2} m$, known as the Holevo bound [4].

The previous approach to demonstrate nonclassicality of temporal quantum correlations is the so-called "temporal Bell inequalities" [5-14]. One of the problems with this approach, stated in [13], is that the classical assumptions behind the temporal inequalities, which are realism and noninvasiveness, were originally chosen to test the quantumness of a temporal evolution of macroscopic quantum systems [5]. As such, they do not provide a convincing test of quantumness in the case of a single evolving system. Moreover, the operational meaning of the assumptions themselves is still a subject of debate [7,11,15].

The fact that in the sequential scenario the evolving system carries information has significant consequences. Let us first consider the simplest possible case: a single two-level system which undergoes a sequence of two black-box operations at time instances $t_{1}$ and $t_{2}$. Each black box has an input describing which settings are chosen and an output describing the measurement result. For a quantum implementation in which the black boxes perform projective measurements with the standard state reduction rule, the inputs have the form of unit vectors $\vec{a}\left(t_{1}\right)$ and $\vec{a}\left(t_{2}\right)$, and the outputs read $\alpha\left(t_{1}\right)= \pm 1$ and $\alpha\left(t_{2}\right)= \pm 1$, respectively. It can be verified [6], that the temporal correlation function $\left\langle\alpha\left(t_{1}\right) \alpha\left(t_{2}\right)\right\rangle$ equals $\vec{a}\left(t_{1}\right) \cdot \vec{a}\left(t_{2}\right)$. This is (up to the sign) the correlation function that would be generated by two separated parties that share the singlet state. Moreover, it leads to a maximal violation of the temporal Clauser-Horne-Shimony-Holt inequality [6]. Can we conclude from these two facts that our system gives rise to nonclassical temporal correlations? The answer is negative-instead of a single qubit one can communicate one classical bit which together with black boxes equipped with classically correlated real vectors 
implement the Toner-Bacon 1-bit protocol for simulating the singlet state [16] adapted to the temporal scenario [17].

A similar situation can arise in the case of multipoint correlations. Suppose the evolution begins with an arbitrary qubit state, then any sequence of $n$ projective measurements leads to correlations that factor into pairs of dot products of the consecutive input vectors [6]. Thus the temporal correlations of $n$ projective measurements on a qubit admit a classical simulation essentially using a sequence of Toner-Bacon protocols.

The ability to classically simulate a correlation function is not restricted to a sequence of projective quantum measurements. For example, consider a sequence of three black boxes with two-setting inputs $\phi\left(t_{k}\right)=\{0, \pi / 2\}$ for $k=1,2,3$ and binary outputs $\alpha\left(t_{k}\right)= \pm 1$ and where the inputs fulfill the additional constraint $\sum_{k} \phi\left(t_{k}\right)=\{0, \pi\}$. Let us assume that the correlation function of outputs $\left\langle\alpha\left(t_{1}\right) \alpha\left(t_{2}\right) \alpha\left(t_{3}\right)\right\rangle=\cos \left[\phi\left(t_{1}\right)+\phi\left(t_{2}\right)+\phi\left(t_{3}\right)\right]$. The function $\left\langle\alpha\left(t_{1}\right) \alpha\left(t_{2}\right) \alpha\left(t_{3}\right)\right\rangle$ can be realized by a sequence of two-outcome positive-operator valued measure (POVM) measurements on a single qubit [18]. It is the correlation function corresponding to the Greenberger-HorneZeilinger (GHZ) state that leads to the GHZ paradox [19]. However, as we prove later in the Letter, the entire setup can be simulated by a classical protocol with exactly 1 bit of classical communication. Again, we cannot verify that truly nonclassical correlations have appeared.

Definition of nonclassical temporal correlations.-The above examples show that identifying a violation of temporal Bell inequalities with so-called "entanglement in time" [6] is not always accurate. Indeed, forward signaling of classical information in the sequential scenario leaves a kind of communication loophole: correlation functions which would be considered nonclassical in the spatial setting can be simulated by classical protocols that use a classical communication channel with capacity given by the Holevo bound of the corresponding quantum particle. The communication implicitly involved in a sequential process motivates adapting the concept of effective classical simulability with respect to the communication cost (see Fig. 1).

Definition 1.-The temporal correlation function $E\left(Y_{1}, \ldots, Y_{N} \mid X_{1}, \ldots, X_{N}\right)$ of the $m$-level physical system is nonclassical if all classical algorithms that simulate the function require more than $\log _{2} m$ bits of classical communication at some step of the simulation.

The idea of quantifying the degree of a nonclassicality of a physical process distributed in space or time by the amount of classical resources needed to simulate it has already appeared in many contexts: communication complexity of simulating spatial quantum correlations [16,20-27], memory complexity of simulating contextual effects [28], and memory complexity of simulating unitary evolution $[13,29-31]$.
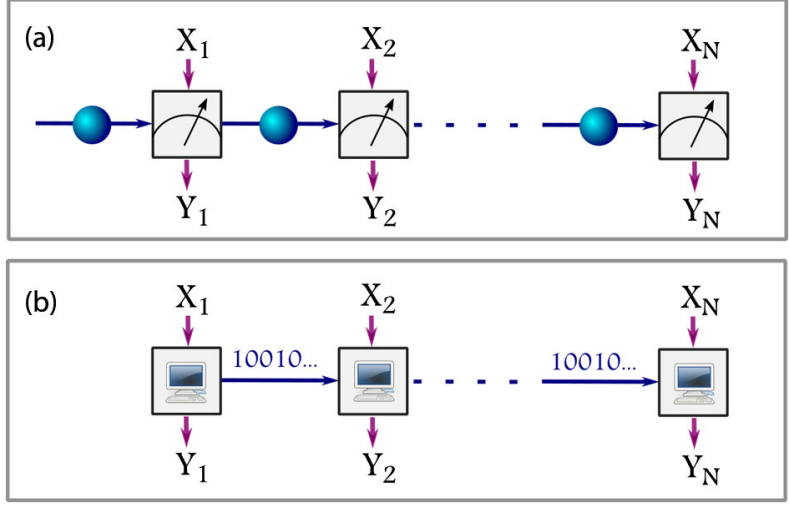

FIG. 1 (color online). Temporal correlation functions. (a) A sequence of $n$ consecutive measurements on a single quantum system with settings provided by inputs $X_{k}$ and outcomes given by numbers $Y_{k}$. We say that temporal correlations are nonclassical if there is no classical simulation depicted in panel (b). At the $i$ th step of the simulation, the $i$ th black box can perform local computations and send classical communication to the next box. The correlation function obtained in scenario (a) is nonclassical if every classical simulation (b) requires more communication than the classical information capacity of the quantum particle in at least one stage of the simulation.

In contrast to previous work, our definition places no restrictions on the operation of the black boxes or the size of their internal memory. We want to decide if a set of correlations is nonclassical, solely on the basis of the correlation function and the number of degrees of freedom of a physical system. The physical implementation (if one exists) which leads to the given correlation function is completely irrelevant from the point of view of our approach. It is a theory-independent definition of nonclassical temporal correlations.

The fact that the simulation of some sequential quantum procedures like contextuality tests or unitary evolution demands resources exceeding the Holevo bound has been already noticed in the literature $[28,30]$. For example, in Ref. [28] the counted resource is the number of internal states of the simulating machines at each stage of the protocol. However, this memory cost can be seen to differ from the information transmitted between each time step of the protocol [32].

We point out that there are other notions of simulability, which can be used to define nonclassicality (e.g., in terms of computational complexity [33]). In this work we solely refer to simulability in the context of communication complexity.

We demonstrate the utility of our definition by presenting a sequence of quantum measurements that give nonclassical temporal correlations provided the number of measurements is sufficiently large. The quantum system and measurements we propose have the appealing property that they are within the reach of current experimental techniques. In the simplest case, we find nonclassical 
temporal correlations for a sequence of 16 POVM measurements on a qubit system.

Nonclassicality of temporal GHZ correlations.Temporal correlations that have the same form as spatial correlations of an $n$-qumit GHZ state:

$$
|\mathrm{GHZ}\rangle=\frac{1}{\sqrt{m}} \sum_{i=1}^{m}|i\rangle^{\otimes n},
$$

are called temporal GHZ correlations. We show that such correlation functions are nonclassical on condition that the number of measurement steps and the number of settings per observer in that sequence is sufficiently large. To prove this fact we utilize modulo- $(m, d)$ games [34], which are distributed computing tasks in the spatial scenario. Modulo- $(m, d)$ games can be solved with certainty with the help of a shared GHZ state (1) but cannot be solved with certainty using classical randomized algorithms, i.e., if the parties are equipped with classically correlated random variables. We translate these games into the sequential scenario and prove that they can always be solved exactly using a sequence of POVM measurements on a single qumit giving rise to temporal GHZ correlations. In Theorem 1, we then show that for some sets of parameters $n, m, d$ they cannot be simulated by any protocol which at each step communicates at most the number of classical bits given by the Holevo bound.

Definition 2.-[Sequential $n$-point modulo- $(m, d)$ problem.] A sequential $n$-point modulo- $(m, d)$ problem is a communication complexity task, in which $n$ separate ordered parties are given $(\log d)$-bit inputs $X_{k}$, with the promise that $\sum_{k=1}^{n} X_{k} \bmod d=0$. The task of the parties is to output values $Y_{k} \in\{0,1, \ldots, m-1\}$ fulfilling $d \sum_{k=1}^{n} Y_{k} \equiv \sum_{k=1}^{n} X_{k} \bmod (m d)$ in a sequential protocol, which at the $k$ th stage allows the $k$ th party to produce her local output $Y_{k}$ and communicate a $c_{k}$-bit message $M_{k}$ to the $(k+1)$ st party.

First, we prove that the above problem can be solved with certainty by a sequence of appropriate generalized quantum measurements on a single qumit. To solve the modulo$(m, d)$ problem in a spatial domain [34], the $n$ parties share state (1) and apply local unitary operations $F_{m}^{\dagger}\left(S_{d m}\right)^{X_{k}}$ to their particles, where $\left[F_{m}\right]_{\alpha \beta}=(1 / \sqrt{m}) \exp (2 i \alpha \beta \pi / m)$ and $\left[S_{d m}\right]_{\alpha \beta}=\exp (2 i \beta \pi / d m) \delta_{\alpha \beta}, \quad \alpha, \beta=0,1, \ldots, m-1$. Finally, the states are locally measured in the standard basis. Let us now find a temporal counterpart. First, note that the bond dimension of the matrix product state representation [35] of the $n$-qumit GHZ state is $m$, which implies that the state can be constructed by a sequential cascade of twoqumit gates $U[36,37]$. This property means that one can map arbitrary local projective measurements in the spatial scenario into a sequence of POVM measurements on a single system with dimension $m$ while keeping the correlation function fixed [18]. Following the construction presented in Ref. [18] one finds the measurement operators $K_{Y_{k}}$ corresponding to different outcomes $Y_{k}$ by solving the system of equations:
$S(U(|\psi\rangle \otimes|0\rangle))=\sum_{Y_{k}=0}^{m-1}\left(K_{Y_{k}}|\psi\rangle\right) \otimes F_{m}^{\dagger}\left(S_{d m}\right)^{X_{j}}\left|Y_{k}\right\rangle$,

where $S$ is a swap operator, and $|\psi\rangle$ is an arbitrary state. For odd dimensions $m$, measurement operators $K_{Y_{k}}$ obtained in this way are diagonal matrices $\mathcal{M}$ with $j$ th diagonal element equal to $[\mathcal{M}(d)]_{j j}=(1 / \sqrt{m}) \exp \left[i X_{k} \pi\left(2^{j}-2\right) / d\right]$. For even $m$, measurement operators corresponding to outputs $Y_{k}$ are $K_{Y_{k}}=\mathcal{A}_{Y_{k}} \mathcal{M}$, where $\mathcal{A}_{Y_{k}}$ is a diagonal matrix with elements \pm 1 and $\pm i$. In particular, for $m=2$, $A_{0}=\operatorname{diag}(1,1), \quad A_{1}=\operatorname{diag}(1,-1) ; \quad$ for $m=4, \quad A_{0}=$ $\operatorname{diag}(1,1,1,1), A_{1}=\operatorname{diag}(1,-i,-1, i), A_{2}=\operatorname{diag}(1,-1$, $1,-1), \quad A_{3}=\operatorname{diag}(1, i,-1,-i) ; \quad$ for $\quad m=6, \quad A_{0}=$ $A_{2}=A_{4}=\operatorname{diag}(1,1,1,1,1,1), \quad A_{1}=A_{3}=A_{5}=\operatorname{diag}(1$, $-1,1,-1,1,-1)$.

We now consider classical simulations of modulo- $(m, d)$ games that use at most $\log m$ bits of classical communication at each step. Before presenting the main result, we first discuss the simplest case $m=d=2$. It turns out that this problem can be simulated with a single bit of communication at each stage (see Sec. I of the Supplemental Material [38]). Since the modulo-(2,2) problem for $n=3$ is equivalent to the scenario of the original GHZ paradox [39], discussed in the introduction, it follows that the two-setting GHZ qubit correlations, which in the spatial domain reveal strong nonclassicality, do not fulfill the definition of nonclassical temporal correlations.

We now provide a general lower bound on the amount of communication which is needed to classically solve these problems, which will imply the main result of our work.

Theorem 1.-Every classical protocol that solves the sequential modulo- $(m, d)$ problem with certainty uses at least $c_{k}=\log (d / m)$ bits of communication in all stages of the protocol except at most $m d-1$ (not necessarily consecutive) stages, when $d$ is an integer power of 2 and $m$ is even.

The proof comes by adapting the argument used in [30] to the scenario of sequential measurements. We provide a stand-alone proof for completeness, since the original proof applies an iterated argument in a slightly informal way, leading to incorrect constants in the analysis.

To perform a proof by contradiction, fix any classical protocol that claims to solve the modulo- $(m, d)$ problem with certainty, while using less than $c_{k}$ bits of communication in some $n_{0}=m d$ stages. We will act as an adversary, constructing two valid inputs of the modulo- $(m, d)$ problem, $X_{k}$ and $X_{k}^{\prime}$, such that $\sum_{k=1}^{n} X_{k} \not \equiv \sum_{k=1}^{n} X_{k}^{\prime} \bmod m d$. The inputs will be defined so that the corresponding outputs $Y_{k}$ and $Y_{k}^{\prime}$ will be indistinguishable, i.e., $Y_{k}=Y_{k}^{\prime}$, for all $1 \leq k \leq n$. Hence, the modulo- $(m, d)$ problem will be solved incorrectly for at least one of the inputs $X_{k}, X_{k}^{\prime}$, leading to a contradiction.

The construction proceeds as follows. Let $K_{0}=$ $\left\{k_{1}, k_{2}, \ldots, k_{n_{0}}\right\}$ be the set of indices of the stages for which the protocol sends messages of size less than $c_{k}$. 
We proceed with the construction of inputs $X_{k}, X_{k}$ ' sequentially, so that the following predicates are fulfilled at any step $k$ : (i) For all $j \leq k, Y_{j}=Y_{j}^{\prime}$. (ii) for all $j \leq k$, $M_{j}=M_{j}^{\prime}$, where $M_{j}$ is the message. The construction of inputs $X_{k}, X_{k}^{\prime}$ proceeds as follows: (i) For any stage $k \notin K_{0}$, $k \neq n$, we set $X_{k}$ arbitrarily, and put $X_{k}^{\prime}=X_{k}$. Clearly, since $M_{k-1}=M_{k-1}^{\prime}$ by the inductive assumption and $X_{k}^{\prime}=X_{k}$, the protocol will act identically in the $k$ th step in both cases; thus, we have $M_{k}=M_{k}{ }^{\prime}$ and $Y_{k}=Y_{k}^{\prime}$. (ii) For any stage $k \in K_{0}$, given message $M_{k-1}=M_{k-1}^{\prime}$ we consider the set of outcome pairs $p(x)=\left(M_{k}(x), Y_{k}(x)\right)$ of the execution $k$ th step of the protocol, taken over all possible inputs $x \in\{0,1, \ldots, d-1\}$. Since $\left|M_{k}\right| \leq 2^{c_{k}}$ for $k \in K_{0}$ and $Y_{k} \in\{0,1, \ldots, m-1\}$, the set of possible output pairs $p(x)$ has less than $2^{c_{k}} m$ elements, where we note that $2^{c_{k}} m=2^{\log (d / m)} m=d$. Consequently, there exists a pair of values $x<x^{\prime}, x, x^{\prime} \in\{0,1, \ldots, d-1\}$, such that $p(x)=$ $p\left(x^{\prime}\right)$. We denote $x^{\prime}=x+\Delta_{k}$, with $\Delta_{k} \in\{1,2, \ldots, d-1\}$. We now put $X_{k}=x$, and choose $X_{k}{ }^{\prime} \in\left\{X_{k}, X_{k}+\Delta_{k}\right\}$, according to a rule which will be described later. Regardless of this choice, we have $M_{k}=M_{k}{ }^{\prime}$ and $Y_{k}=Y_{k}^{\prime}$. (iii) Finally, in stage $k=n$, we set $X_{k}$ so that the input $\left\{X_{k}\right\}_{k=1}^{n}$ satisfies the modulo- $d$ promise, and also put $X_{n}^{\prime}=X_{n}$.

It remains to show that it is possible to fix $X_{k}^{\prime}$ from among each pair of considered values $\{x, x+\Delta\}$ for $k \in K_{0}$, so that $\sum_{k \in K_{0}} X_{k} \not \equiv \sum_{k \in K_{0}} X_{k}^{\prime} \bmod (m d)$. This is possible by the following lemma, whose proof is presented in Sec. II of the Supplemental Material [38].

Lemma 1.-Let $\left\{\Delta_{k}\right\}_{k \in K_{0}}$ be any sequence of integers, with $\Delta_{k} \in\{1,2, \ldots, m d-1\}$, where $\left|K_{0}\right|=n_{0} \geq m d, m$ is even and $d=2^{s}$ for some integer $s>0$. Then, there exists a subset of indices $K_{0}^{\prime} \subseteq K_{0}$ such that $\sum_{k \in K_{0}^{\prime}} \Delta_{k} \equiv 0 \bmod d$ and $\sum_{k \in K_{0}^{\prime}} \Delta_{k} \not \equiv 0 \bmod (m d)$.

Using Lemma 1, we then pick $X_{k}^{\prime}=X_{k}+\Delta_{k}$ for all steps $k \in K_{0}^{\prime}$, and put $X_{k}^{\prime}=X_{k}$ for all steps $k \in K_{0} \backslash K_{0}^{\prime}$. This completes our construction.

In the above proof, we restricted our considerations to deterministic protocols. For randomized protocols, the claim of the proposition also holds in the following sense: any randomized protocol which does not satisfy the assumptions of the proposition will lead to an incorrect output for some instances of the modulo- $(m, d)$ problem, with strictly positive probability.

The above theorem can be treated as a temporal version of Bell inequalities with auxiliary communication in the spatial scenario $[40,41]$, and directly leads to the main result of our work:

Proposition 1.-The temporal GHZ correlations arising from sequential measurements on a single qumit, where $m$ is even, are nonclassical for $n \geq 2 m^{3}$.

Proof.-It suffices to show that there exists a modulo$(m, d)$ game for some $d$ and $n$, for which classical simulation uses in at least one stage of the protocol more than $\log m$ bits of communication. Using Theorem 1, we need to choose parameters so that the following two conditions are fulfilled: (i) $\log (d / m)>\log m$, which means that the classical communication needed is greater than the Holevo bound for the system. (ii) $n \geq m d$, which guarantees that the amount of communication equal to $\log (d / m)>\log m$ bits is needed in at least one stage.

Now, let $d$ be the smallest integer power of 2 larger than $m^{2}$, we have $d \leq 2 m^{2}$. Taking any $n \geq 2 m^{3} \geq m d$, guarantees that the numbers $m, n, d$ fulfill both of the above conditions.

The above proposition shows temporal GHZ correlations for systems of even dimensions reveal temporal nonclassicality if $n$ is sufficiently large. Any classical simulation uses more bits of communication than the Holevo bound not only in at least one stage of the protocol (as demands Definition 1), but in almost all stages (for detailed analysis, see Sec. III in the Supplemental Material [38]).

As Proposition 1 holds also for a single qubit case it demonstrates for the first time that the simulation of a temporal correlation function on a qubit system demands resources that exceed the Holevo bound. A similar effect for the unitary evolution of a single qubit was shown in Ref. [30].

Conclusions.-In general terms, correlations between physical systems can be considered in two distinct scenarios: spatial and temporal. In the spatial scenario local measurements are performed by spacelike separated parties who may share a source of joint randomness but who are unable to communicate. Temporal correlations, on the other hand, arise from a sequence of measurements on a single physical system at different time instances. Communication is now allowed from one time instance to the next but is limited by the information capacity of the system.

We showed that in the temporal measurement scenario one can define a notion of nonclassical $n$-point correlations with a clear operational interpretation. Namely, such correlations cannot be simulated by any classical protocol whose communication is limited by the Holevo capacity of the evolving quantum system. In addition, we demonstrated that the temporal analogue of generalized GHZ correlations arising from sequential measurements on a single qumit, reveal nonclassicality in the temporal scenario provided the number of measurements is large enough. Apart from these foundational issues, we provided the first general lower bound on the communication complexity of simulating multipoint quantum correlations in a sequential measurement scenario (see Ref. [27] for results on the classical communication cost of simulating spatial GHZ correlations).

We would like to thank Marcin Pawłowski, Rafał Demkowicz-Dobrzański, and Marek Żukowski for helpful discussions and the anonymous referees for their constructive engagement that improved the presentation of the results. A.K. was supported by the ANR, project DISPLEXITY. M. M. and A.P. gratefully acknowledge the financial support by Polish Ministry of Science and Higher Education Grant No. IdP2011 000361. This work is 
supported by the National Research Foundation, Ministry of Education of Singapore Grant No. RG98/13, NCN Grant No. 2014/14/M/ST2/0081, and by the EC under the FP7 IP project SIQS cofinanced by the Polish Ministry of Science and Higher Education.

*marcinm495@gmail.com

[1] J. Bell, Physics 1, 195 (1964).

[2] J. Clauser, M. Horne, A. Shimony, and R. Holt, Phys. Rev. Lett. 23, 880 (1969).

[3] N. Brunner, D. Cavalcanti, S. Pironio, V. Scarani, and S. Wehner, Rev. Mod. Phys. 86, 419 (2014).

[4] A. Holevo, Probl. Inf. Transm. 9, 177 (1973).

[5] A. J. Leggett and A. Garg, Phys. Rev. Lett. 54, 857 (1985).

[6] Č. Brukner, S. Taylor, S. Cheung, and V. Vedral, arXiv: quant-ph/0402127.

[7] R. Lapiedra, Europhys. Lett. 75, 202 (2006).

[8] M. Barbieri, Phys. Rev. A 80, 034102 (2009).

[9] D. Avis, P. Hayden, and M. M. Wilde, Phys. Rev. A 82, 030102(R) (2010).

[10] T. Fritz, New J. Phys. 12, 083055 (2010).

[11] M. Markiewicz, P. Kurzynski, J. Thompson, S.-Y. Lee, A. Soeda, T. Paterek, and D. Kaszlikowski, Phys. Rev. A 89, 042109 (2014).

[12] C. Budroni, T. Moroder, M. Kleinmann, and O. Gühne, Phys. Rev. Lett. 111, 020403 (2013).

[13] M. Żukowski, Front. Phys. 9, 629 (2014).

[14] C. Budroni and C. Emary, Phys. Rev. Lett. 113, 050401 (2014).

[15] O. J. Maroney and C. G. Timpson, arXiv:1412.6139.

[16] B. F. Toner and D. Bacon, Phys. Rev. Lett. 91, 187904 (2003).

[17] Since Toner-Bacon protocol [16] is a sequential two-stage protocol with one-way communication, it can as well be seen as a protocol which simulates two-point temporal correlations.

[18] M. Markiewicz, A. Przysiężna, S. Brierley, and T. Paterek, Phys. Rev. A 89, 062319 (2014).

[19] D. Greenberger, M. Horne, A. Shimony, and A. Zeilinger, Am. J. Phys. 58, 1131 (1990).

[20] G. Brassard, R. Cleve, and A. Tapp, Phys. Rev. Lett. 83, 1874 (1999).

[21] S. Massar, D. Bacon, N. Cerf, and R. Cleve, Phys. Rev. A 63, 052305 (2001).

[22] O. Regev and B. Toner, in Proceedings of the 48th Annual IEEE Symposium on Foundations of Computer Science (IEEE, New York, 2007), p. 384.

[23] J. Degorre, S. Laplante, and J. Roland, Phys. Rev. A 75, 012309 (2007).

[24] Y. Shi and Y. Zhu, SIAM J. Comput. 38, 753 (2008).
[25] T. Vertesi and E. Bene, Phys. Rev. A 80, 062316 (2009).

[26] A. Kosowski and M. Markiewicz, arXiv:1310.2217.

[27] G. Brassard, L. Devroye, and C. Gravel, arXiv:1303.5942.

[28] M. Kleinmann, O. Gühne, J. R. Portillo, J.-A. Larsson, and A. Cabello, New J. Phys. 13, 113011 (2013).

[29] P. Trojek, C. Schmid, M. Bourennane, C. Brukner, M. Żukowski, and H. Weinfurter, Phys. Rev. A 72, 050305(R) (2005).

[30] E. F. Galvão and L. Hardy, Phys. Rev. Lett. 90, 087902 (2003).

[31] A. Montina, Phys. Rev. A 77, 022104 (2008).

[32] Kleinmann et al. [28] discuss correlations between a class of quantum measurements relevant to contextuality tests and consider the memory cost of simulating a sequence of compatible measurements. In Ref. [28] "memory" refers to the total number of states in a classical simulation rather than the communication between measurements. This difference can be seen in the example of the Peres-Mermin contextuality game [see Eq. (8) and Theorem 2 in [28] ]. Kleinmann et al. show that the memory cost of any simulation is $\log (4)$ as a total of four states are necessary and sufficient to simulate the Peres-Mermin contextuality game. However, Eq. (8) in [28] also shows that each state only needs to change into one of three possible states at each time step; thus the communication complexity is upper bounded by $\log (3)$.

[33] M. Oszmaniec, J. Gutt, and M. Kuś, Phys. Rev. A 90, 020302(R) (2014).

[34] M. Boyer, arXiv:quant-ph/0408090.

[35] D. Perez-Garcia, F. Verstraete, M. M. Wolf, and J. I. Cirac, Quantum Inf. Comput. 7, 401 (2007).

[36] C. Schön, E. Solano, F. Verstraete, J. I. Cirac, and M. M. Wolf, Phys. Rev. Lett. 95, 110503 (2005).

[37] M.-C. Bañuls, D. Perez-Garcia, M. M. Wolf, F. Verstraete, and J. I. Cirac, Phys. Rev. A 77, 052306 (2008).

[38] See Supplemental Material at http://link.aps.org/ supplemental/10.1103/PhysRevLett.115.120404 for a discussion of a communication complexity of the sequential modulo-(2,2) problem, detailed proof of a technical Lemma 1 and a brief discussion of communication complexity properties of sequential protocols simulating GHZ (Greenberger-Horne-Zeilinger) correlations.

[39] C. Gavoille, A. Kosowski, and M. Markiewicz, Lect. Notes Comput. Sci. 5805, 243 (2009).

[40] D. Bacon and B. F. Toner, Phys. Rev. Lett. 90, 157904 (2003).

[41] K. Maxwell and E. Chitambar, Phys. Rev. A 89, 042108 (2014). 